\title{
Impact of Screw Type on Kyphotic Deformity Correction after Spine Fracture Fixation: Cannulated versus Solid Pedicle Screw
}

\author{
Mahmood Ali Arbash, Ashik Mohsin Parambathkandi, Abdul Moeen Baco, Abduljabbar Alhammoud \\ Department of Orthopedics, Hamad Medical Corporation, Doha, Qatar
}

\section{Study Design: Retrospective review.}

Purpose: To detect the effect of cannulated (poly-axial head) and solid (mono-axial head) screws on the local kyphotic angle, vertebral body height, and superior and inferior angles between the screw and the rod in the surgical management of thoracolumbar fractures.

Overview of Literature: Biomechanics studies showed that the ultimate load, yield strength, and cycles to failure were significantly lower with cannulated (poly-axial head) pedicle comparing to solid core (mono-axial head).

Methods: The medical charts of patients with thoracolumbar fractures who underwent pedicle screw fixation with cannulated or solid pedicle screws were retrospectively reviewed; the subjects were followed up from January 2011 to December 2015.

Results: Total 178 patients (average age, 36.1 12.4 years; men, 142 [84.3\%]; women, 28 [15.7\%]) with thoracolumbar fractures who underwent surgery and were followed up at Hamad Medical Corporation were classified, based on the screw type as those with cannulated screws and those with solid screws. The most commonly affected level was L1, followed by L2 and D12. Surgical correction of the local kyphotic angle was significantly different in the groups; however, there was no significant difference in the loss of correction of the local kyphotic angle of the groups. Surgical correction of the reduction in the vertebral body height showed statistical significance, while the average loss of correction in the reduction of the vertebral body height was not significantly different. The measurement of the angles made by the screws on the rods was not significantly different between the cannulated (poly-axial head) and solid (mono-axial head) screw groups.

Conclusions: Solid screws were superior in terms of providing increased correction of the kyphotic angle and height of the fractured vertebra than the cannulated screws; however, no difference was noted between the screws in the maintenance of the superior and inferior angles of the screw with the rod.

Keywords: Solid (mono-axial head) screw; Cannulated (poly-axial head) screw; Thoracolumbar fractures; Kyphotic angle

\section{Introduction}

Traumatic spine fractures are common injuries that have several causes, particularly falls from a height and road traffic accidents. If not treated properly, they can cause major disabilities, and approximately $12 \%$ of the patients who presented to the trauma unit in the emergency department of the Hamad Medical Corporation are classi-

Received Dec 9, 2017; Revised Apr 12, 2018; Accepted May 22, 2018

Corresponding author: Abduljabbar Alhammoud

Department of Orthopedics, Hamad Medical Corporation, Doha, Qatar

Tel: +974-33273574, Fax: +974-44392608, E-mail: aghammoud85@hotmail.com 
fied in this category [1].

For unstable spine fractures, fixation is necessary; it is accomplished by either the open traditional technique or the minimally invasive surgery (MIS) technique that is gaining popularity among spine surgeons because of the lower risk of blood loss, decreased operation time, and lesser postoperative pain [2,3]. Cannulated (poly-axial head) pedicle screws (CS) that use a guide wire for screw insertion are considered the cornerstone of the MIS technique, while in the open technique, the use of solid core (mono-axial head) screws (SCS) remains a valid option [4-8] (Figs. 1, 2).

To our knowledge, several studies have investigated the physical characteristics of CS an SCS, such as bending performance, static and dynamic load to failure, and pullout strength, using biomechanical tests on cadaveric or constructed modules. However, few trials have assessed the radiological or clinical differences between these screws in non-English literature [4,5,9-12].

We aimed to compare the correction of the local kyphotic angle and the vertebral body height between the two types of screws and assess their effect on the superior and inferior angles of the screw with the rod.

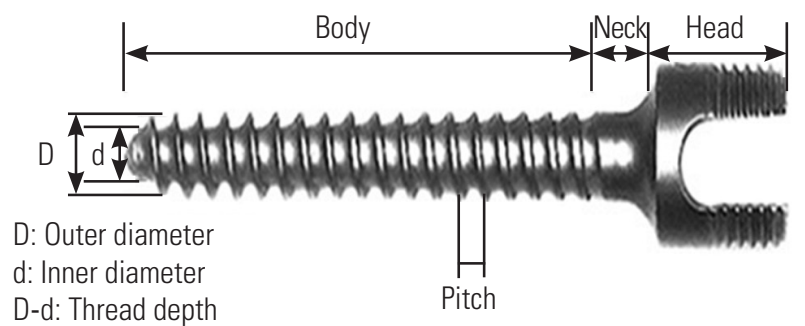

Fig. 1. Solid (mono-axial head) screw $[6,7]$.

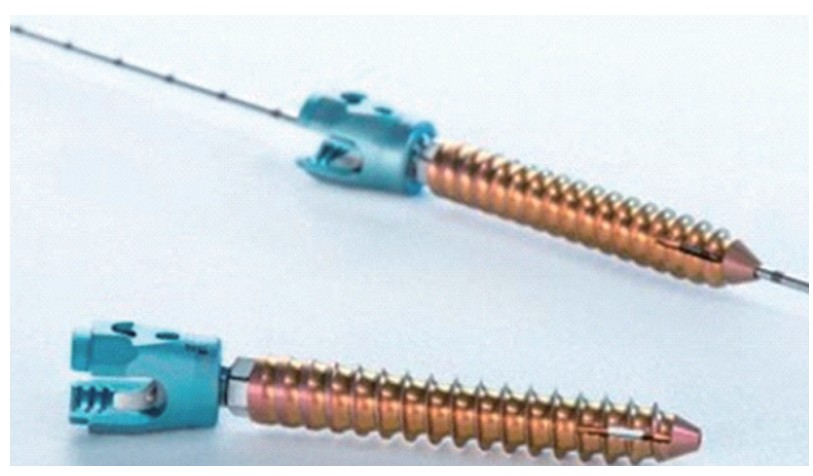

Fig. 2. Cannulated (poly-axial head) screw [8].

\section{Materials and Methods}

A retrospective review of the medical charts of all patients with thoracolumbar fractures who underwent pedicle screw fixation with CS or SCS was performed, and these patients were followed up at the orthopedic spine department from January 2011 to December 2015 after obtaining approval from the medical research center.

Data regarding the general demographic characteristics (age and sex), comorbidities, injury characteristics (mode of trauma, level of injury, and fracture classification), surgery-related parameters (open versus MIS, CS versus SCS screw), and radiological parameters (superior and inferior angles between the screw and the connecting rod, local
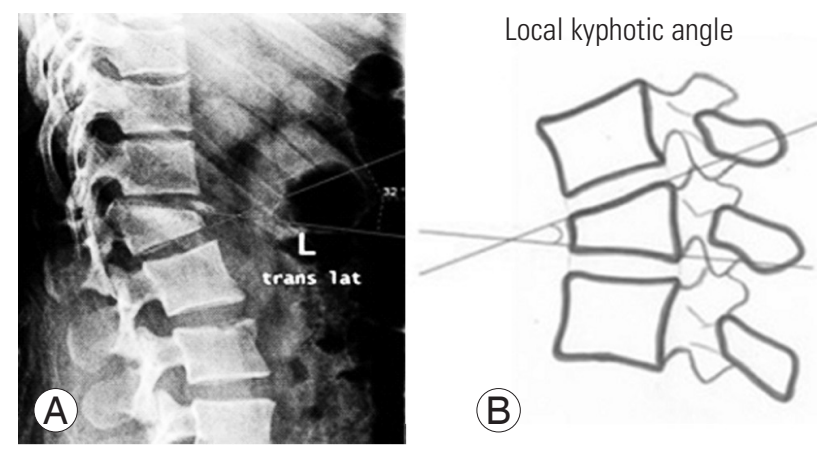

Fig. 3. (A, B) Local kyphotic angle [13]

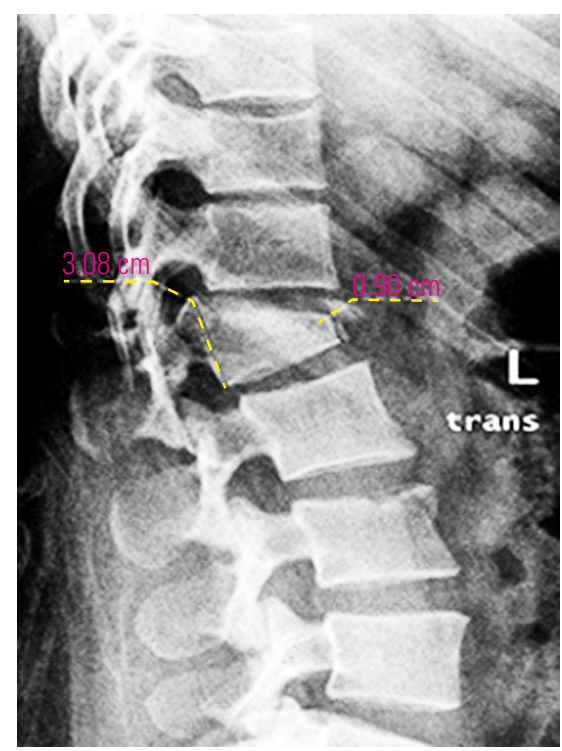

Fig. 4. Vertebral body height [9]. Reduction of height was calculated by dividing the anterior wall height over the posterior wall height and then multiply by 100 , this give us the percentage of the height from actual height of the fractured vertebra, and by subtracting it from 100 we get the percentage of reduction in height. So for this case, $\{(0.90)$ $3.08) \times 100\}-100=70.77 \%$ is the reduction in height. 
kyphotic angle, and vertebral body high) were collected at different follow-up intervals (preoperative, postoperative, and at 3,6, and 9-12 months postoperatively).

The local kyphotic angle is the angulation between the superior and inferior plates of the fractured vertebra [13] (Fig. 3). The vertebral body height (the percentage of height from the original height) was calculated by dividing the anterior wall height by the posterior wall height and then multiplying it with 100 [9] (Fig. 4). The superior angle is the angle between the rod and the superior screw, while the inferior angle is the angle between the rod and the inferior screw (two angles proposed by the research team to show the relationship between the rod and the

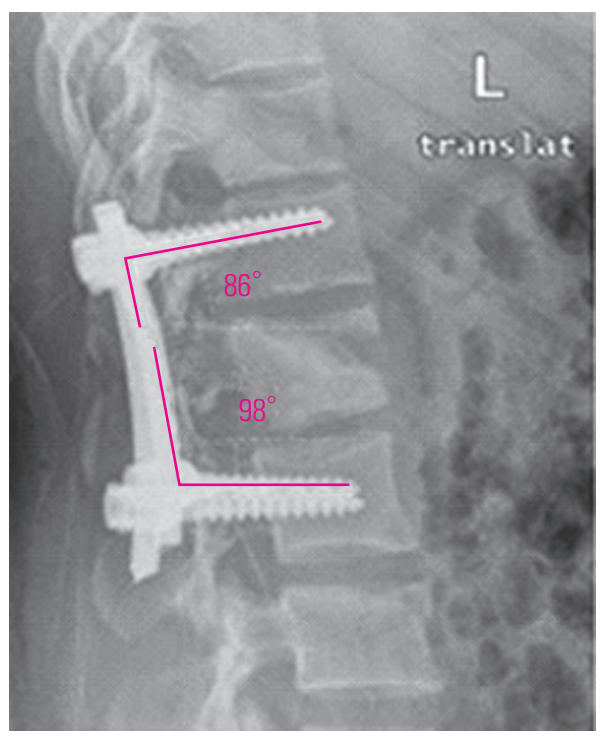

Fig. 5. Superior and inferior angle. screw) (Fig. 5).

Indications for surgery were an increase $>30^{\circ}$ in the kyphotic angle, loss of $>50 \%$ in the vertebral body height, or neurological deficit. The choice of open or MIS surgery was made by the surgeon, based on his/her preference and experience, while the choice between CS and SCS was dependent on availability.

Frequency (percentage) and mean \pm standard deviation or median and range values are used to represent the categorical and continuous values, as appropriate. Descriptive statistics were used to summarize the demographic characteristics, injury characteristics, surgery-related parameters, and radiological parameters. Fisher exact test and chi-square test were used to evaluate the associations among two or more qualitative variables, while unpaired $t$-test and Mann-Whitney $U$-tests were used for the quantitative data. A 2 -sided $p$-value $<0.05$ was considered statistically significant. All the statistical analyses used IBM SPSS ver. 22.0 (IBM Corp., Armonk, NY, USA) and Epi Info 2000 (Centers for Disease Control and Prevention, Atlanta, GA, USA).

All the data were collected after ethical approval (approval no., 16158/16) was obtained from the Medical Research Center, the Hamad Medical Corporation, Doha, Qatar. Waived informed consent was taken for this retrospective study.

\section{Results}

Total 178 patients (average age, $36.1 \pm 12.4$ years; men, 142 [84.3\%]; women, 28 [15.7\%]) with thoracolumbar frac-

Table 1. Demographic data

\begin{tabular}{|c|c|c|c|c|}
\hline Characteristic & Total & Cannulated & Solid & $p$-value \\
\hline No. of patients & 178 & $100(56.2)$ & $78(43.8)$ & \\
\hline Age (yr) & $36.1 \pm 12.4$ & $37.8 \pm 14$ & $34.3 \pm 9.9$ & 0.067 \\
\hline Sex & & & & 0.473 \\
\hline Male & 150 (84.3) & 86 (57.3) & $64(42.7)$ & \\
\hline Female & $28(15.7)$ & $14(50.0)$ & $14(50.0)$ & \\
\hline Technique & & & & 0.001 \\
\hline Open & 110 (61.8) & $32(29.1)$ & 78 (70.9) & \\
\hline Minimally invasive surgery & $68(38.2)$ & $68(100.0)$ & 0 & \\
\hline \multicolumn{5}{|l|}{ Level } \\
\hline Thoracic spine & $52(29.2)$ & - & - & \\
\hline Lumber spine & $126(70.8)$ & - & - & \\
\hline
\end{tabular}

Values are presented as number (\%) or mean \pm standard deviation. 
Table 2. Radiological data during follow-up

\begin{tabular}{|c|c|c|c|c|}
\hline Variable & Cannulated & Solid & Confidence interval & $p$-value \\
\hline \multicolumn{5}{|l|}{ Local kyphotic angle $\left({ }^{\circ}\right)$} \\
\hline Preoperative & $17.4 \pm 8.9$ & $20.1 \pm 10.7$ & -0.16 to 5.7 & 0.065 \\
\hline Postoperative & $8.6 \pm 6.9$ & $7.1 \pm 5.6$ & -3.4 to 0.38 & 0.116 \\
\hline Follow-up 1 & $11.5 \pm 6.6$ & $9.2 \pm 6.7$ & -4.40 to -0.04 & 0.046 \\
\hline Follow-up 2 & $10.5 \pm 6.2$ & $8.3 \pm 6.0$ & -4.92 to 0.51 & 0.111 \\
\hline Follow-up 3 & $11.6 \pm 6.4$ & $7.5 \pm 6.0$ & -11.34 to 3.23 & 0.249 \\
\hline Between preoperative and postoperative & $8.84 \pm 10.4$ & $13.0 \pm 11.2$ & 0.86 to 7.44 & 0.014 \\
\hline Between postoperative and final follow-up & $4.2 \pm 5.9$ & $9.5 \pm 5.5$ & -1.54 to 12.25 & 0.117 \\
\hline \multicolumn{5}{|l|}{ Vertebral body height (\%) } \\
\hline Preoperative & $39.6 \pm 28.2$ & $41.5 \pm 27.6$ & & \\
\hline Postoperative & $23.5 \pm 13.3$ & $20.1 \pm 12.6$ & -7.3 to 9.47 & 0.085 \\
\hline Follow-up 1 & $29.4 \pm 14.2$ & $21.4 \pm 13.7$ & -12.6 to -3.4 & 0.001 \\
\hline Follow-up 2 & $28.4 \pm 12.4$ & $20.0 \pm 13.2$ & -14.1 to -2.7 & 0.004 \\
\hline Follow-up 3 & $26.0 \pm 15.1$ & $20.1 \pm 15.5$ & -24.1 to 12.3 & 0.498 \\
\hline Between preoperative and postoperative & $16.1 \pm 14.9$ & $21.4 \pm 15.0$ & 97.0 to 9.9 & 0.024 \\
\hline Between postop and final follow-up & $18.0 \pm 13.0$ & $20.2 \pm 7.1$ & -9.3 to 13.7 & 0.682 \\
\hline \multicolumn{5}{|l|}{ Superior angle $\left({ }^{\circ}\right)$} \\
\hline Intraoperative & $80.8 \pm 6.3$ & $87.1 \pm 4.9$ & 4.2 to 8.3 & 0.001 \\
\hline Postoperative & $80.8 \pm 5.7$ & $88.4 \pm 5.4$ & 5.9 to 9.2 & 0.001 \\
\hline Follow-up 1 & $79.7 \pm 5.8$ & $86.7 \pm 8.2$ & 4.7 to 9.3 & 0.001 \\
\hline Follow-up 2 & $79.8 \pm 6.9$ & $88.7 \pm 4.8$ & 6.1 to 11.5 & 0.001 \\
\hline Follow-up 3 & $77.75 \pm 4.3$ & $90.1 \pm 6.5$ & 4.3 to 20.3 & 0.006 \\
\hline Between postoperative and final follow-up & $4.5 \pm 0.7$ & $0.3 \pm 6.0$ & -15.8 to 6.1 & 0.324 \\
\hline \multicolumn{5}{|l|}{ Inferior angle $\left({ }^{\circ}\right)$} \\
\hline Intraoperative & $93.8 \pm 5.8$ & $95.6 \pm 5.0$ & -0.1 to 3.7 & 0.069 \\
\hline Postoperative & $93.1 \pm 5.0$ & $94.1 \pm 4.9$ & -0.5 to 2.5 & 0.196 \\
\hline Follow-up 1 & $91.6 \pm 5.2$ & $92.8 \pm 4.2$ & -0.3 to 2.5 & 0.125 \\
\hline Follow-up 2 & $92.03 \pm 4.0$ & $93.4 \pm 3.7$ & -0.5 to 2.8 & 0.165 \\
\hline Follow-up 3 & $96.0 \pm 3.0$ & $91.3 \pm 4.2$ & - 10.1 to 0.8 & 0.092 \\
\hline Between postoperative and final follow-up & $1.5 \pm 4.9$ & $0.8 \pm 3.5$ & -9 to 7.6 & 0.838 \\
\hline
\end{tabular}

tures who underwent surgery and were followed up at the Hamad Medical Corporation were analyzed. The most commonly affected level was L1 in $37.1 \%$ of the patients, followed by L2 (in 18\%) and D12 (in 17.7\%) (Table 1).

The average preoperative, postoperative, and final follow-up local kyphotic angles of the fractured vertebra were $17.4^{\circ} \pm 8.9^{\circ}, 8.6^{\circ} \pm 6.9^{\circ}$, and $11.6^{\circ} \pm 6.4^{\circ}$, respectively, in the CS group, while these were $20.1^{\circ} \pm 10.7^{\circ}, 7.1^{\circ} \pm 5.6^{\circ}$, and $7.5^{\circ} \pm 6^{\circ}$, respectively, in the SCS group. Surgical correction (i.e., the difference between the preoperative and postoperative local kyphotic angles) was significantly different between the CS group $\left(8.8^{\circ} \pm 10.4^{\circ}\right)$ and the SCS group $\left(13^{\circ} \pm 11.2^{\circ}, p=0.014\right)$. In contrast, the average loss of correction (i.e., the difference between the final follow- 

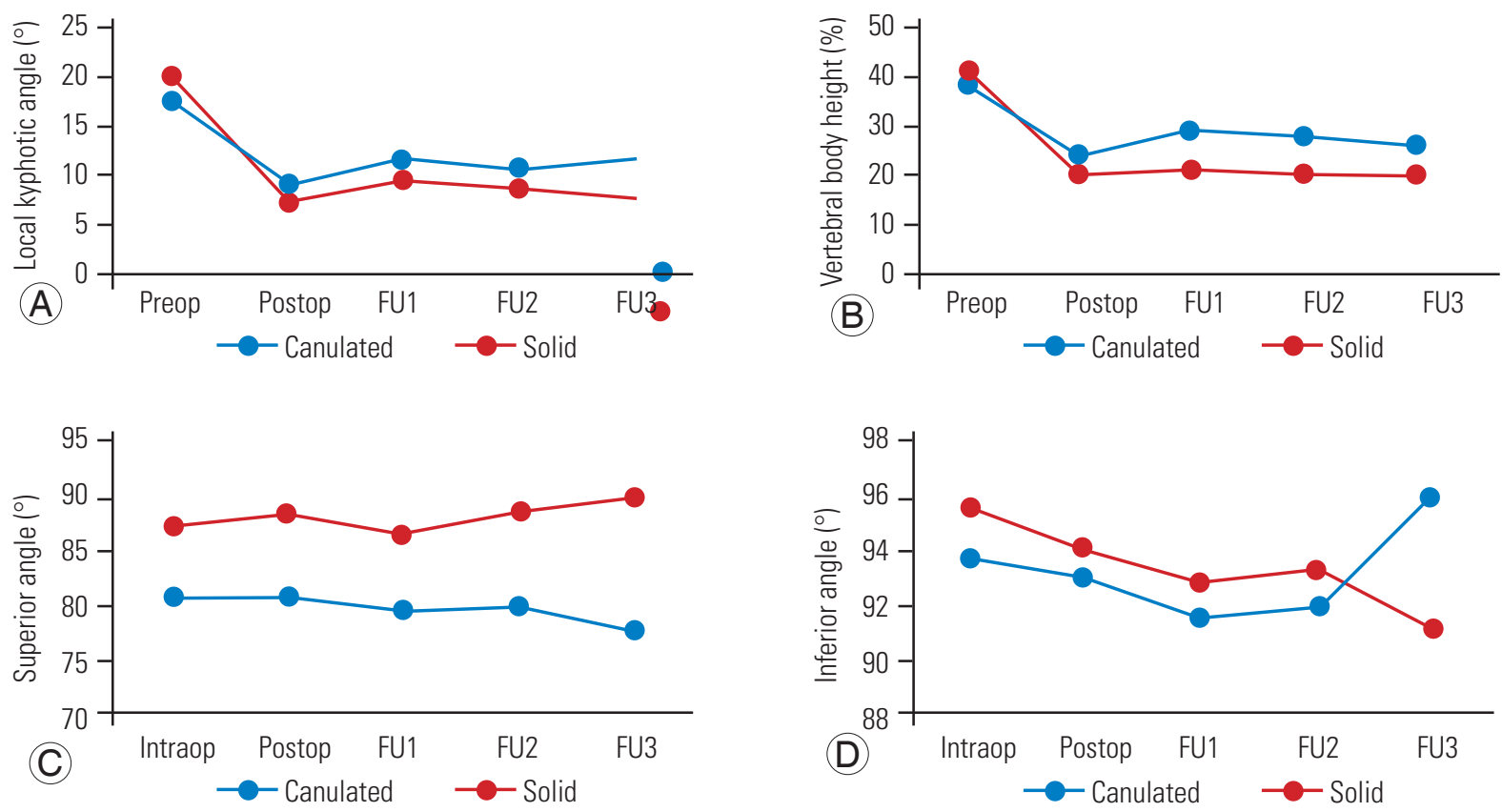

Fig. 6. Changes in the local kyphotic angle (A), vertebral body height (B), superior angle (C), and inferior angle (D) during follow-up. Preop, preoperative; Postop, postoperative; FU, follow-up; Intraop, intraoperative.

up and the postoperative local kyphotic angles) was not significantly different between the CS group $\left(4.2^{\circ} \pm 5.9^{\circ}\right)$ and the SCS group $\left(9.5^{\circ} \pm 5.5^{\circ}, p=0.117\right)$ (Table 2, Fig. 6).

The average intraoperative, postoperative, and final follow-up reductions in the vertebral body height of the fractured vertebra were $39.6 \% \pm 28.2 \%, 23.5 \% \pm 13.3 \%$, and $26 \% \pm 15.1 \%$, respectively, in the CS group, and $41.5 \% \pm 27.6 \%, 20.1 \% \pm 12.6 \%$, and $20.1 \% \pm 15.5 \%$, respectively, in the SCS group. The surgical correction of the reduction of the vertebral body height was significantly different between the CS $(16.1 \% \pm 14.9 \%)$ and SCS $(21.4 \% \pm 15 \%)$ groups $(p=0.024)$. However, the average loss of correction of the reduction in the vertebral body height was $18 \% \pm 13 \%$ and $20.2 \% \pm 7.1 \%$ in the CS and SCS groups, respectively; this difference was not statistically significant ( $p=0.682$ ) (Table 2, Fig. 6).

The difference between the postoperative and final follow-up superior and inferior angles was not statistically significant ( $p=0.324$ and $p=0.838$, respectively), with an average superior angle of $4.5^{\circ} \pm 0.7^{\circ}$ and $-0.3^{\circ} \pm 6^{\circ}$ in the CS and SCS groups, respectively, and an average inferior angle of $1.5^{\circ} \pm 4.9^{\circ}$ and $0.8^{\circ} \pm 3.5^{\circ}$ in the CS and SCS groups, respectively (Table 2, Fig. 6).

\section{Discussion}

Thoracolumbar fractures are common in Qatar and add a significant burden to the healthcare system. Qatar is a developing country with a rapidly growing population and infrastructure. The current data from the Hamad Medical Corporation, the main tertiary-care hospital in Qatar, show that annually, about 200 patients present with traumatic spinal injuries primarily attributed to motor vehicle accidents and falls from a height, making traumatic spinal fracture the leading cause of disability in this population [1].

A literature review showed no differences in the vertebral body height local kyphotic angle correction between the open and MIS techniques; however, the latter had a shorter operative time and less blood loss [2,3]. To our knowledge, this is the first study to compare the effect of CS and SCS on the local kyphotic angle and vertebral body height after spine fracture fixation. Many studies have compared the biomechanics of CS and SCS to show that the ultimate load, yield strength, and cycles to failure were significantly lower with the CS than with the SCS $[4,5,10,11]$.

Another study performed biomechanical tests to compare the bending performance of the two types of screws and found that the latter has significantly poorer bending 
performance [9]. Other studies compared the effect of CS versus that of SCS on the stability of the construct after fixation, and they showed that incorporating a CS did not significantly decrease the construct's stiffness [12].

The superior and inferior angles reflect the bending of the screw and impending failure and broken. Our study revealed changes in the superior angle during follow-up, with statistically significant differences between the CS and SCS in the first $(p=0.001)$, second $(p=0.001)$, and final follow-ups ( $p=0.006$ ); however, no significant difference was observed between the postoperative and final followup $(p=0.324)$. No statistically significant difference was detected in the inferior angle during the first, second, and final follow-ups ( $p=0.125, p=0.165$, and $p=0.092$, respectively) or in the superior angle between postoperative and final follow-up ( $p=0.838$ ) (Table 2).

We use both techniques in our hospital; generally, we use CS in MIS techniques and SCS for the open technique (Table 2). One of the most important limitations of the current study is the lack of correlation of the radiological findings with the functional outcome and complication rate. Another limitation is the relatively short follow-up duration; thus, long-term follow-up studies are warranted to evaluate the long-term complications and failure of both types of screws.

\section{Conclusions}

SCS were found to provide superior correction of the kyphotic angle and height of the fractured vertebra than cannulated screws. However, no difference was noted between the screws with respect to the maintenance of the superior and inferior angles of the screw with the rod.

\section{Conflict of Interest}

No potential conflict of interest relevant to this article was reported.

\section{Acknowledgments}

This work was supported by the Hamad Medical Corporation Medical Research Center.

\section{References}

1. El-Faramawy A, El-Menyar A, Zarour A, et al. Pre- sentation and outcome of traumatic spinal fractures. J Emerg Trauma Shock 2012;5:316-20.

2. McAnany SJ, Overley SC, Kim JS, Baird EO, Qureshi SA, Anderson PA. Open versus minimally invasive fixation techniques for thoracolumbar trauma: a meta-analysis. Global Spine J 2016;6:186-94.

3. Wang H, Zhou Y, Li C, Liu J, Xiang L. Comparison of open versus percutaneous pedicle screw fixation using the sextant system in the treatment of traumatic thoracolumbar fractures. Clin Spine Surg 2017;30:E239-46.

4. Dundon JM, Gould GC, Herbenick MA, Hamilton JA. Cannulated screw with solid core insert: stronger than cannulated screws. Am J Orthop (Belle Mead NJ) 2014;43:E191-3.

5. Shepard MF, Davies MR, Abayan A, Kabo JM, Wang JC. Effects of polyaxial pedicle screws on lumbar construct rigidity. J Spinal Disord Tech 2002;15:2336.

6. Cho W, Cho SK, Wu C. The biomechanics of pedicle screw-based instrumentation. J Bone Joint Surg Br 2010;92:1061-5.

7. Weise L, Suess O, Picht T, Kombos T. Transpedicular screw fixation in the thoracic and lumbar spine with a novel cannulated polyaxial screw system. Med Devices (Auckl) 2008;1:33-9.

8. Medical Expo. Cannulated pedicle screw/polyaxial: S4(R) element augmentation [Internet]. [place unknown]: Medical Expo; 2016 [cited 2017 Dec 1]. Available from: http://www.medicalexpo.com.

9. Shih KS, Hsu CC, Hou SM, Yu SC, Liaw CK. Comparison of the bending performance of solid and cannulated spinal pedicle screws using finite element analyses and biomechanical tests. Med Eng Phys 2015;37:879-84.

10. Stanford RE, Loefler AH, Stanford PM, Walsh WR. Multiaxial pedicle screw designs: static and dynamic mechanical testing. Spine (Phila Pa 1976) 2004;29:367-75.

11. Chen LH, Tai CL, Lai PL, et al. Pullout strength for cannulated pedicle screws with bone cement augmentation in severely osteoporotic bone: influences of radial hole and pilot hole tapping. Clin Biomech (Bristol, Avon) 2009;24:613-8.

12. Fogel GR, Reitman CA, Liu W, Esses SI. Physical characteristics of polyaxial-headed pedicle screws and biomechanical comparison of load with their 
failure. Spine (Phila Pa 1976) 2003;28:470-3.

13. Son KH, Chung NS, Jeon CH. Measurement of vertebral compression and kyphosis in the thoracolum- bar and lumbar fractures. J Korean Soc Spine Surg 2010;17:120-6. 